\title{
An EPQ Model with Two-Component Demand under Fuzzy Environment and Weibull Distribution Deterioration with Shortages
}

\author{
S. Sarkar and T. Chakrabarti \\ Department of Applied Mathematics, University of Calcutta, 92 APC Road, Kolkata 700009, India \\ Correspondence should be addressed to S. Sarkar, sanchita771@rediffmail.com
}

Received 26 April 2011; Revised 28 June 2011; Accepted 15 July 2011

Academic Editor: Hsien-Chung Wu

Copyright (C) 2012 S. Sarkar and T. Chakrabarti. This is an open access article distributed under the Creative Commons Attribution License, which permits unrestricted use, distribution, and reproduction in any medium, provided the original work is properly cited.

\begin{abstract}
A single-item economic production model is developed in which inventory is depleted not only due to demand but also by deterioration. The rate of deterioration is taken to be time dependent, and the time to deterioration is assumed to follow a two-parameter Weibull distribution. The Weibull distribution, which is capable of representing constant, increasing, and decreasing rates of deterioration, is used to represent the distribution of the time to deterioration. In many reallife situations it is not possible to have a single rate of production throughout the production period. Items are produced at different rates during subperiods so as to meet various constraints that arise due to change in demand pattern, market fluctuations, and so forth. This paper models such a situation. Here it is assumed that demand rate is uncertain in fuzzy sense, that is, it is imprecise in nature and so demand rate is taken as triangular fuzzy number. Then by using $\alpha$-cut for defuzzification the total variable cost per unit time is derived. Therefore the problem is reduced to crisp average costs. The multiobjective model is solved by Global Criteria method with the help of GRG (Generalized Reduced Gradient) Technique. In this model shortages are permitted and fully backordered. Numerical examples are given to illustrate the solution procedure of the two models.
\end{abstract}

\section{Introduction}

The classical EOQ (Economic Order Quantity) inventory models were developed under the assumption of constant demand. Later many researchers developed EOQ models taking linearly increasing or decreasing demand. Donaldson [1] discussed for the first time the classical no-shortage inventory policy for the case of a linear, positive trend in demand. Wagner and Whitin [2] developed a discrete version of the problem. Silver and Meal [3] formulated an approximate solution procedure as "Silver Meal heuristic" for 
a deterministic time-dependent demand pattern. Mitra et al. [4] extended the model to accommodate a demand pattern having increasing and decreasing linear trends. Deb and Chaudhuri [5] extended for the first time the inventory replenishment policy with linear trend to accommodate shortages. After some correction in the above model [5], Dave [6] applied Silver's [7] heuristic to it incorporating shortages. Researchers have also worked on inventory models with time-dependent demand and deterioration. Models by Dave and Patel [8], Sachan [9], Bahari-Kashani [10], Goswami and Chaudhuri [11], and Hariga [12] all belong to this category. In addition to these demand patterns, some researchers use ramp type demand. Ramp type demand is one in which demand increases up to a certain time after which it stabilizes and becomes constant. Ramp type demand precisely depicts the demand of the items, such as newly launched fashion goods and cosmetics, garments, and automobiles for which demand increases as they are launched into the market and after some time it becomes constant. Today most of the real-world decisionmaking problems in economic, technical, and environmental ones are such that the inventory related demands are not deterministic but imprecise in nature. Furthermore in real-life problems the parameters of the stochastic inventory models are also fuzzy, that is, not deterministic in nature and this is the case of application of fuzzy probability in the inventory models.

In 1965, the first publication in fuzzy set theory by Zadeh [13] showed the intention to accommodate uncertainty in the nonstochastic sense rather than the presence of random variables. After that fuzzy set theory has been applied in many fields including productionrelated areas. In the 1990s, several scholars began to develop models for inventory problems under fuzzy environment. Park [14] and Ishii and Konno [15] discussed the case of fuzzy cost coefficients. Roy and Maiti [16] developed a fuzzy economic order quantity (EOQ) model with a constraint of fuzzy storage capacity. Chang and Yao [17] solved the economic reorder point with fuzzy backorders.

Some inventory problems with fuzzy shortage cost are analyzed by Katagiri and Ishii [18]. A unified approach to fuzzy random variables is considered by Krätschmer [19]; Kao and Hsu [20] discussed a single-period inventory model with fuzzy demand. Fergany and EIWakeel [21] considered the probabilistic single-item inventory problem with varying order cost under two linear constraints. Hala and EI-Saadani [22] analysed constrained singleperiod stochastic uniform inventory model with continuous distribution of demand and varying holding cost. Fuzzy models for single-period inventory problem were discussed by Li et al. [23]. Banerjee and Roy [24] considered application of the Intuitionistic Fuzzy Optimization in the constrained Multiobjective Stochastic Inventory Model. Banerjee and Roy [25] also discussed the single- and multiobjective stochastic inventory model in fuzzy environment. Lee and Yao [26] and Chang and Yao [17] investigated the economic production quantity model, and Lee and Yao [27] studied the EOQ model with fuzzy demands. A common characteristic of these studies is that shortages are backordered without extra costs.

Buckley [28] introduced a new approach and applications of fuzzy probability and after that Buckley and Eslami [29-31] contributed three remarkable articles about uncertain probabilities. Hwang and Yao [32] discussed the independent fuzzy random variables and their applications. Formalization of fuzzy random variables is considered by Colubi et al. [33]. Krätschmer [19] analyzed a unified approach to fuzzy random variables. Luhandjula [34] discussed a mathematical programming in the presence of fuzzy quantities and random variables. 
Many researchers developed inventory models in which both the demand and deteriorating rate are constant. Although the constant demand assumption helps to simplify the problem, it is far from the actual situation where demand is always in change. In order to make research more practical, many researchers have studied other forms of demand. Among them time-dependant demand has attracted considerable attention. In this paper exponentially increasing demand has been considered which is a general form of linear and nonlinear time-dependent demand. Various types of uncertainties and imprecision are inherent in real inventory problems. They are classically modeled using the approaches from the probability theory. However there are uncertainties that cannot be appropriately treated by usual probabilistic models. The questions how to define inventory optimization task in such environment and how to interpret optimal solution arise. This paper considers the modification of EPQ formula in the presence of imprecisely estimated parameters with fuzzy demands where backorders are permitted, yet a shortage cost is incurred. The demand rate is taken as triangular fuzzy number. Since the demand is fuzzy the average cost associated with inventory is fuzzy in nature. So the average cost in fuzzy sense is derived. The fuzzy model is defuzzified by using $\alpha$-cut of fuzzy number. This multiobjective problem is solved by Global Criteria method with the help of GRG (Generalized Reduced Gradient) technique, and it is illustrated with the help of numerical example.

\section{Preliminaries}

For developing the mathematical model we are to introduce certain preliminary definitions and results which will be used later on.

Definition 2.1 (fuzzy number). A fuzzy subset $\widetilde{A}$ of real number $R$ with membership function $\mu_{\tilde{A}}: R \rightarrow[0,1]$ is called a fuzzy number if

(a) $\tilde{A}$ is normal, that is, there exists an element $x_{0}$ such that $\mu_{\tilde{A}}\left(x_{0}\right)=1$ is normal, that is, there exists an element $x_{0}$ such that $\mu_{\tilde{A}}\left(x_{0}\right)=1$;

(b) $\tilde{A}$ is convex, that is, $\mu_{\tilde{A}}\left(\lambda x_{1}+(1-\lambda) x_{2}\right) \geq \mu_{\tilde{A}}\left(x_{1}\right) \wedge \mu_{\tilde{A}}\left(x_{2}\right)$ for all $x_{1}, x_{2} \in R$ and $\lambda \in[0,1]$

(c) $\mu_{\widetilde{A}}$ is upper semicontinuous;

(d) $\operatorname{supp}(\widetilde{A})$ is bounded, here $\operatorname{supp}(\widetilde{A})=\operatorname{supp}\left\{x \in R: \mu_{\tilde{A}}(x)>0\right\}$.

Definition 2.2 (triangular fuzzy number). A Triangular Fuzzy number (TFN) $\tilde{A}$ is specified by the triplet $\left(a_{1}, a_{2}, a_{3}\right)$ and is defined by its continuous membership function $\mu_{\tilde{A}}(x)$, a continuous mapping $\mu_{\tilde{A}}: R \rightarrow[0,1]$ as follows:

$$
\mu_{\tilde{A}}(x)= \begin{cases}\frac{x-a_{1}}{a_{2}-a_{1}} & \text { if } a_{1} \leq x<a_{2} \\ \frac{a_{3}-x}{a_{3}-a_{2}} & \text { if } a_{2} \leq x \leq a_{3} \\ 0 & \text { otherwise. }\end{cases}
$$


Definition 2.3 (integration of a fuzzy function). Let $\tilde{f}(x)$ be a fuzzy function from $[a, b] \subseteq R$ to $R$ such that $\tilde{f}(x)$ is a fuzzy number, that is, a piecewise continuous normalized fuzzy set on $R$. Then integral of any continuous $\alpha$-level curve of $\tilde{f}(x)$ over $[a, b]$ always exists and the integral of $\tilde{f}(x)$ over $[a, b]$ is then defined to be the fuzzy set

$$
\tilde{I}(a, b)=\left\{\left(\int_{a}^{b} f^{-}(\alpha)(x) d x+\int_{a}^{b} f^{+}(\alpha)(x) d x, \alpha\right)\right\} .
$$

The determination of the integral $\widetilde{I}(a, b)$ becomes somewhat easier if the fuzzy function is assumed to be $L R$ type. We will therefore assume that $\tilde{f}(x)=\left(f_{1}(x), f_{2}(x), f_{3}(x)\right)_{L R}$ is a fuzzy number in $L R$ representation for all $x \subseteq[a, b]$, where $f_{1}(x), f_{2}(x)$, and $f_{3}(x)$ are assumed to be positive integrable functions on $[a, b]$. Dubois and Prade have shown that under these conditions

$$
\tilde{I}(a, b)=\left(\int_{a}^{b} f_{1}(x) d x, \int_{a}^{b} f_{2}(x) d x, \int_{a}^{b} f_{3}(x) d x\right)_{L R} .
$$

Definition 2.4 ( $\alpha$-cut of fuzzy number). The $\alpha$-cut of a fuzzy number is a crisp set which is defined as $[\tilde{A}]_{\alpha}=\left\{x \in R: \mu_{\tilde{A}}(x) \geq \alpha\right\}$. According to the definition of fuzzy number it is seen that $\alpha$-cut is a nonempty bounded closed interval; it can be denoted by

$$
[\tilde{A}]_{\alpha}=\left[A_{L}(\alpha), A_{R}(\alpha)\right]
$$

$A_{L}(\alpha)$ and $A_{R}(\alpha)$ are the lower and upper bounds of the closed interval, where

$$
\begin{aligned}
& {[\tilde{A}]_{L}(\alpha)=\inf \left\{x \in R: \mu_{\tilde{A}}(x) \geq \alpha\right\},} \\
& {[\tilde{A}]_{R}(\alpha)=\sup \left\{x \in R: \mu_{\tilde{A}}(x) \geq \alpha\right\} .}
\end{aligned}
$$

Definition 2.5 (Global Criteria method (Rao,nd)). In the global criteria method $x^{*}$ is found by minimizing a preselected global criteria, $F(X)$, such as the sum of the squares of the relative deviations of the individual objective functions from the feasible ideal solutions. The $X^{*}$ is found by minimizing

$$
F(X)=\sum_{i=1}^{k}\left\{\frac{f_{i}\left(X_{i}^{*}\right)-f_{i}(X)}{f_{i}\left(X_{i}^{*}\right)}\right\}^{p}
$$

$$
\text { subject to } g_{j}(X) \leq 0, \quad j=1,2, \ldots, m \text {, }
$$


where $p$ is a constant (as usual value of $p$ is 2) and $X_{i}^{*}$ is the ideal solution for the $i$ th objective function. The solution $X_{i}^{*}$ is obtained by minimizing $f_{i}(X)$ subject to the constraints $g_{j}(X) \leq$ $0, j=1,2, \ldots, m$.

\section{Notations and Assumptions}

\subsection{Notations}

(i) $T$ is the length of one cycle.

(ii) $I(t)$ is the inventory level at time $t$.

(iii) $\tilde{R}(t)$ is the induced fuzzy demand around $R$ which is a function of time.

(iv) $K_{1}$ and $K_{2}$ are two production rates varies with demand.

(v) $C_{1}$ is the holding cost per unit time.

(vi) $C_{2}$ is the shortage cost per unit time.

(vii) $C_{3}$ is the unit purchase cost.

(viii) $C_{4}$ is the fixed ordering cost of inventory.

(ix) $T$ is the cycle time.

(x) GC is the global criteria.

(xi) $\theta$ is the deterioration rate of finished items.

\subsection{Assumptions}

(i) The demand function $\tilde{R}(t)$ is taken to be fuzzy function of time, that is, $\widetilde{R}(t)=\tilde{a} e^{b t}$.

(ii) $K_{1}$ and $K_{2}$ are two demand depended production variables, that is, $K_{1}=\beta_{1} \tilde{R}(t)$ and $K_{2}=\beta_{2} \tilde{R}(t)$, where $\beta_{1}$ and $\beta_{2}$ are constants.

(iii) Replenishment is instantaneous.

(iv) Lead time (i.e., the length between making of a decision to replenish an item and its actual addition to stock) is assumed to be zero. The assumption is made so that the period of shortage is not affected.

(v) The rate of deterioration at any time $t>0$ follows the two-parameter Weibull distribution: $\theta(t)=\gamma \beta t^{\beta-1}$, where $\gamma(0<\gamma<1)$ is the scale parameter and $\beta(>0)$ is the shape parameter. The implication of the Weibull rate (two parameter) is that the items in inventory start deteriorating the instant they are received into inventory. The rate of deterioration-time relationship is shown in Figure 1. 


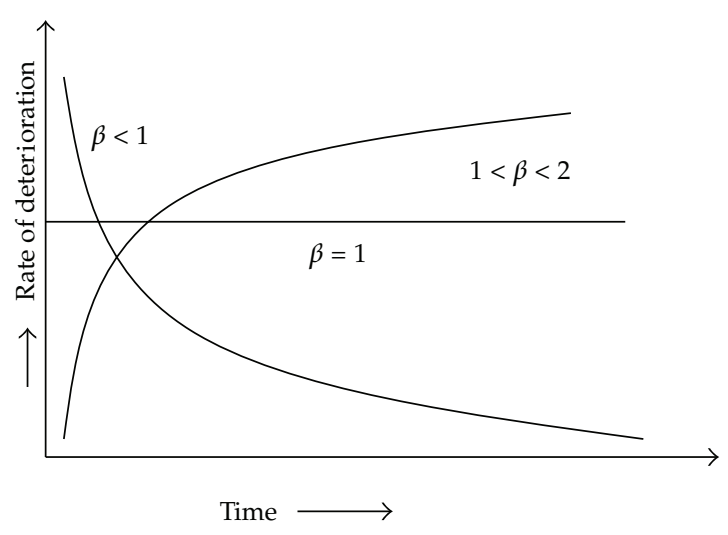

Figure 1

When $\beta=1, z(t)=\alpha$ (a constant) which is the case of exponential decay.

When $\beta<1$ rate of deterioration is decreasing with time.

When $\beta>1$, rate of deterioration is increasing with time.

(vi) Shortages are allowed and are fully backlogged.

\section{Mathematical Modeling and Analysis}

Here we assume that the production starts at time $t=0$ at the rate $K_{1}$ and the stock attains a level $P$ at $t=z$. Then at $t=z$ the production rate changes to $K_{2}$ and continues up to $t=y$, where the inventory level reaches the maximum level $Q$. And the production stops at $t=y$ and the inventory gradually depletes to zero at $t=t_{1}$ mainly to meet the demands and partly for deterioration. Now shortages occur and accumulate to the level $S$ at time $t=t_{2}$. The production starts again at a rate $K_{1}$ at $t=t_{2}$ and the backlog is cleared at time $t=T$ when the stock is again zero. The cycle then repeats itself after time $T$.

The model is represented by Figure 2 .

The changes in the inventory level can be described by the following differential equations:

$$
\begin{gathered}
\frac{d \tilde{I}(t)}{d t}+\gamma \beta t^{\beta-1} \tilde{I}(t)=\left(\beta_{1}-1\right) \tilde{a} e^{b t} \quad 0 \leq t \leq z, \\
\frac{d \tilde{I}(t)}{d t}+\gamma \beta t^{\beta-1} \tilde{I}(t)=\left(\beta_{2}-1\right) \tilde{a} e^{b t} \quad z \leq t \leq y, \\
\frac{d \tilde{I}(t)}{d t}+\gamma \beta t^{\beta-1} \tilde{I}(t)=-\tilde{a} e^{b t} \quad y \leq t \leq t_{1}, \\
\frac{d \tilde{I}(t)}{d t}=-\tilde{a} e^{b t} \quad t_{1} \leq t \leq t_{2}, \\
\frac{d \tilde{I}(t)}{d t}=-\left(\beta_{1}-1\right) \tilde{a} e^{b t} \quad t_{2} \leq t \leq T
\end{gathered}
$$

with initial conditions $I(z)=P, I(y)=Q, I\left(t_{1}\right)=0, I\left(t_{2}\right)=S$, and $I(T)=0$. 


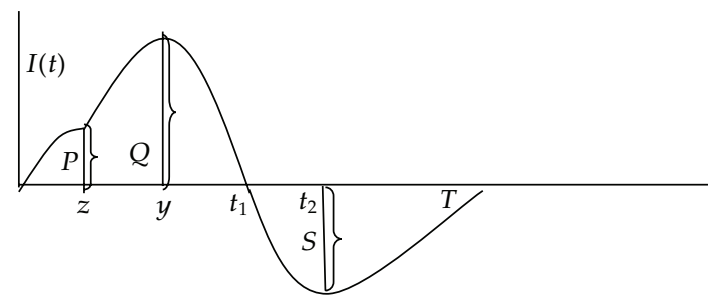

Figure 2

The differential equations (4.1) are fuzzy differential equations. To solve this differential equation at first we take the $\alpha$-cut then the differential equations reduces to

$$
\begin{aligned}
& \frac{d I_{1}^{+}(t)}{d t}+\gamma \beta t^{\beta-1} I_{1}^{+}(t)=\left(\beta_{1}-1\right) a^{+}(\alpha) e^{b t}, \quad 0 \leq t \leq z, \\
& \frac{d I_{1}^{-}(t)}{d t}+\gamma \beta t^{\beta-1} I_{1}^{-}(t)=\left(\beta_{1}-1\right) a^{-}(\alpha) e^{b t}, \quad 0 \leq t \leq z, \\
& \frac{d I_{2}^{+}(t)}{d t}+\gamma \beta t^{\beta-1} I_{2}^{+}(t)=\left(\beta_{2}-1\right) a^{+}(\alpha) e^{b t}, \quad z \leq t \leq y, \\
& \frac{d I_{2}^{-}(t)}{d t}+\gamma \beta t^{\beta-1} I_{2}^{-}(t)=\left(\beta_{2}-1\right) a^{-}(\alpha) e^{b t}, \quad z \leq t \leq y, \\
& \frac{d I_{3}^{+}(t)}{d t}+\gamma \beta t^{\beta-1} I_{3}^{+}(t)=-a^{-}(\alpha) e^{b t}, \quad y \leq t \leq t_{1}, \\
& \frac{d I_{3}^{-}(t)}{d t}+\gamma \beta t^{\beta-1} I_{3}^{-}(t)=-a^{+}(\alpha) e^{b t}, \quad y \leq t \leq t_{1}, \\
& \frac{d I_{4}^{+}(t)}{d t}=-a^{-}(\alpha) e^{b t}, \quad t_{1} \leq t \leq t_{2}, \\
& \frac{d I_{4}^{-}(t)}{d t}=-a^{+}(\alpha) e^{b t}, \quad t_{1} \leq t \leq t_{2}, \\
& \frac{d I_{5}^{+}(t)}{d t}=-\left(\beta_{1}-1\right) a^{-}(\alpha) e^{b t}, \quad t_{2} \leq t \leq T, \\
& \frac{d I_{5}^{-}(t)}{d t}=-\left(\beta_{1}-1\right) a^{+}(\alpha) e^{b t}, \quad t_{2} \leq t \leq T,
\end{aligned}
$$


where

$$
\begin{aligned}
& I_{i}^{+}=\sup \left\{x \in R: \mu_{\widetilde{I}_{i}}(x) \geq \alpha\right\}, \quad i=1,2,3,4,5, \\
& I_{i}^{-}=\inf \left\{x \in R: \mu_{\widetilde{I}_{i}}(x) \geq \alpha\right\}, \quad i=1,2,3,4,5 .
\end{aligned}
$$

Similarly $a^{+}(\alpha), a^{-}(\alpha)$ have usual meaning.

The solutions of the differential equations (4.2) to (4.11) are, respectively, represented by

$$
\begin{aligned}
I_{1}^{+}(t)= & \left(\frac{\left(\beta_{1}-1\right)}{b}\right)\left(e^{b t}-1\right)\left\{a_{3}-\alpha\left(a_{3}-a_{2}\right)\right\}-\frac{\gamma \beta t^{\beta+1}\left(\beta_{1}-1\right) a_{3}}{(1+\beta)}, \quad 0 \leq t \leq z, \\
I_{1}^{-}(t)= & \left(\frac{\left(\beta_{1}-1\right)}{b}\right)\left(e^{b t}-1\right)\left\{a_{1}+\alpha\left(a_{2}-a_{1}\right)\right\}-\frac{\gamma \beta t^{\beta+1}\left(\beta_{1}-1\right) a_{1}}{(1+\beta)}, \quad 0 \leq t \leq z, \\
I_{2}^{+}(t)= & P\left(1+\gamma z^{\beta}-\gamma t^{\beta}\right)+\left(\beta_{2}-1\right) \frac{\left\{a_{3}-\alpha\left(a_{3}-a_{2}\right)\right\}\left(e^{b t}-e^{b z}\right)}{b}-\frac{\gamma t^{\beta}\left(\beta_{2}-1\right) a_{3}}{b}\left(e^{b t}-e^{b z}\right) \\
& +\gamma\left\{a_{3}-\alpha\left(a_{3}-a_{2}\right)\right\} \frac{\left(\beta_{2}-1\right)}{b} t^{\beta}\left(e^{b t}-1\right)-\gamma\left\{a_{3}-\alpha\left(a_{3}-a_{2}\right)\right\} \frac{\left(\beta_{2}-1\right) z^{\beta}\left(e^{b z}-1\right)}{b} \\
& +\left\{a_{3}-\alpha\left(a_{3}-a_{2}\right)\right\} \frac{\gamma \beta\left(\beta_{2}-1\right)}{(1+\beta)}\left(z^{\beta+1}-t^{\beta+1}\right), \quad z \leq t \leq y, \\
I_{2}^{-}(t)= & P\left(1+\gamma z^{\beta}-\gamma t^{\beta}\right)+\left(\beta_{2}-1\right) \frac{\left\{a_{1}+\alpha\left(a_{2}-a_{1}\right)\right\}\left(e^{b t}-e^{b z}\right)}{b}-\frac{\gamma t^{\beta}\left(\beta_{2}-1\right) a_{1}}{b}\left(e^{b t}-e^{b z}\right) \\
& +\left\{a_{1}+\alpha\left(a_{2}-a_{1}\right)\right\} \frac{\gamma\left(\beta_{2}-1\right)}{b} t^{\beta}\left(e^{b t}-1\right)-\left\{a_{1}+\alpha\left(a_{2}-a_{1}\right)\right\} \frac{\gamma\left(\beta_{2}-1\right) z^{\beta}\left(e^{b z}-1\right)}{b} \\
& -\left\{a_{1}+\alpha\left(a_{2}-a_{1}\right)\right\} \gamma \beta \frac{\left(t_{1}^{\beta+1}-t^{\beta+1}\right)}{(1+\beta)}, y \leq t \leq t_{1}, \\
& +\left\{a_{1}+\alpha\left(a_{2}-a_{1}\right)\right\} \frac{\beta \gamma\left(\beta_{2}-1\right)}{(1+\beta)}\left(z^{\beta+1}-t^{\beta+1}\right), \quad z \leq t \leq y, \\
I_{3}^{+}(t)= & {\left[\frac{\left\{a_{1}+\alpha\left(a_{2}-a_{1}\right)\right\}}{b}\right]\left(e^{b t_{1}}-e^{b t}\right)-\left(\frac{a_{1} \gamma t^{\beta}}{b}\right)\left(e^{b t_{1}}-e^{b t}\right) } \\
& \left.+\alpha\left(a_{2}-a_{1}\right)\right\} \\
b\left\{t_{1}^{\beta}\left(e^{b t_{1}}-1\right)-t^{\beta}\left(e^{b t}-1\right)\right\} & \\
b & \\
&
\end{aligned}
$$




$$
\begin{aligned}
I_{3}^{-}(t)= & {\left[\frac{\left\{a_{3}-\alpha\left(a_{3}-a_{2}\right)\right\}}{b}\right]\left(e^{b t_{1}}-e^{b t}\right)-\left(\frac{a_{3} \gamma t^{\beta}}{b}\right)\left(e^{b t_{1}}-e^{b t}\right) } \\
& +\left[\frac{\left\{a_{3}-\alpha\left(a_{3}-a_{2}\right)\right\}}{b}\right] \gamma\left\{t_{1}^{\beta}\left(e^{b t_{1}}-1\right)-t^{\beta}\left(e^{b t}-1\right)\right\} \\
& -\left\{a_{3}-\alpha\left(a_{3}-a_{2}\right)\right\} \gamma \beta \frac{\left(t_{1}^{\beta+1}-t^{\beta+1}\right)}{(1+\beta)}, \quad y \leq t \leq t_{1}, \\
I_{4}^{+}(t)= & -\frac{\left\{a_{1}+\alpha\left(a_{2}-a_{1}\right)\right\}\left(e^{b t}-e^{b t_{1}}\right)}{b}, \quad t_{1} \leq t \leq t_{2}, \\
I_{4}^{-}(t)= & -\frac{\left\{a_{3}-\alpha\left(a_{3}-a_{2}\right)\right\}\left(e^{b t}-e^{b t_{1}}\right)}{b}, \quad t_{1} \leq t \leq t_{2}, \\
I_{5}^{+}(t)= & \frac{\left(\beta_{1}-1\right)\left\{a_{1}+\alpha\left(a_{2}-a_{1}\right)\right\}\left(e^{b T}-e^{b t}\right)}{b}, \quad t_{2} \leq t \leq T, \\
I_{5}^{-}(t)= & \frac{\left(\beta_{1}-1\right)\left\{a_{3}-\alpha\left(a_{3}-a_{2}\right)\right\}\left(e^{b T}-e^{b t}\right)}{b}, \quad t_{2} \leq t \leq T .
\end{aligned}
$$

Therefore the upper $\alpha$-cut of fuzzy stock holding cost is given by

$$
\begin{aligned}
H C^{+} & =\left(\frac{C_{1}}{T}\right)\left[\int_{0}^{z} I_{1}^{+}(t) d t+\int_{z}^{\alpha_{1} t_{1}} I_{2}^{+}(t) d t+\int_{\alpha_{1} t_{1}}^{t_{1}} I_{3}^{+}(t) d t\right] \\
& =\left(\frac{C_{1}}{T}\right)\left[A_{1}+B_{1} t_{1}+C_{1} t_{1}^{\beta+1}+D_{1} t_{1}^{\beta+2}+E_{1} t_{1}^{\beta+3}+F_{1} e^{b t_{1}} t_{1}+G_{1} e^{b t_{1}} t_{1}^{\beta+1}+H_{1} e^{b \alpha_{1} t_{1}}+I_{1} e^{b t_{1}}\right] .
\end{aligned}
$$

Calculations are shown in Appendix A.

And the lower $\alpha$-cut of fuzzy stock holding cost is given by

$$
\begin{aligned}
H C^{-} & =\left(\frac{C_{1}}{T}\right)\left[\int_{0}^{z} I_{1}^{-}(t) d t+\int_{z}^{\alpha_{1} t_{1}} I_{2}^{-}(t) d t+\int_{\alpha_{1} t_{1}}^{t_{1}} I_{3}^{-}(t) d t\right] \\
& =\left(\frac{C_{1}}{T}\right)\left[A_{2}+B_{2} t_{1}+C_{2} t_{1}^{\beta+1}+D_{2} t_{1}^{\beta+2}+E_{2} t_{1}^{\beta+3}+F_{2} e^{b t_{1}} t_{1}+G_{2} e^{b t_{1}} t_{1}^{\beta+1}+H_{2} e^{b \alpha_{1} t_{1}}+I_{2} e^{b t_{1}}\right] .
\end{aligned}
$$

Calculations are shown in Appendix B.

(1) As demand is fuzzy in nature shortage cost is also fuzzy in nature. 
Therefore the upper $\alpha$-cut of shortage cost is given by

$$
\begin{aligned}
\mathrm{S.C}^{+}= & \frac{C_{2}}{T}\left\{\int_{t_{1}}^{\eta T} I_{4}^{+}(t) d t+\int_{\eta T}^{T} I_{5}^{+}(t) d t\right\} \\
= & \frac{C_{2}}{T} \frac{\left\{a_{1}+\alpha\left(a_{2}-a_{1}\right)\right\}}{b}\left\{\frac{-e^{b \eta T}}{b}+\eta T e^{b t_{1}}+\frac{e^{b t_{1}}}{b}-t_{1} e^{b t_{1}}\right\} \\
& +\frac{\mathrm{C}_{2}}{\mathrm{~T}} \frac{\left\{a_{1}+\alpha\left(a_{2}-a_{1}\right)\right\}\left(\beta_{1}-1\right)}{b^{2}}\left\{e^{b T} T-\frac{e^{b T}}{b}-\eta T e^{b T}+\frac{e^{b \eta T}}{b}\right\} .
\end{aligned}
$$

Also the lower $\alpha$-cut of shortage cost is given by

$$
\begin{aligned}
\mathrm{S.C}^{-}= & \frac{C_{2}}{T}\left\{\int_{t_{1}}^{\eta T} I_{4}^{-}(t) d t+\int_{\eta T}^{T} I_{5}^{-}(t) d t\right\} \\
= & \frac{C_{2}}{T} \frac{\left\{a_{3}-\alpha\left(a_{3}-a_{2}\right)\right\}}{b}\left\{\frac{-e^{b \eta T}}{b}+\eta T e^{b t_{1}}+\frac{e^{b t_{1}}}{b}-t_{1} e^{b t_{1}}\right\} \\
& +\frac{C_{2}}{T} \frac{\left\{a_{3}-\alpha\left(a_{3}-a_{2}\right)\right\}\left(\beta_{1}-1\right)}{b^{2}}\left\{e^{b T} T-\frac{e^{b T}}{b}-\eta T e^{b T}+\frac{e^{b \eta T}}{b}\right\} .
\end{aligned}
$$

(2) Annual cost due to deteriorated unit is also fuzzy as demand is fuzzy quantity.

Therefore deterioration cost per year is given by

$$
\begin{aligned}
\text { D.C } & =\frac{C_{3}}{T}\left\{\int_{0}^{z}\left(K_{1}-R\right) d t+\int_{z}^{\alpha_{1} t_{1}}\left(K_{2}-R\right) d t-\int_{\alpha_{1} t_{1}}^{t_{1}} R d t\right\} \\
& =\frac{C_{3}}{T}\left\{\int_{0}^{z} \tilde{a}\left(\beta_{1}-1\right) e^{b t} d t+\int_{z}^{\alpha_{1} t_{1}} \tilde{a}\left(\beta_{2}-1\right) e^{b t} d t-\int_{\alpha_{1} t_{1}}^{t_{1}} \tilde{a} e^{b t} d t\right\} \\
& =\frac{C_{3}}{T}\left\{\int_{0}^{z} f_{1}(t) f_{2}(t) f_{3}(t) d t+\int_{z}^{\alpha_{1} t_{1}} \phi_{1}(t) \phi_{2}(t) \phi_{3}(t) d t-\int_{\alpha_{1} t_{1}}^{t_{1}} \varphi_{1}(t) \varphi_{2}(t) \varphi_{3}(t) d t\right\},
\end{aligned}
$$

where

$$
\begin{gathered}
f_{1}(t)=a_{1}\left(\beta_{1}-1\right) e^{b t}, \quad f_{2}(t)=a_{2}\left(\beta_{1}-1\right) e^{b t}, \quad f_{3}(t)=a_{3}\left(\beta_{1}-1\right) e^{b t}, \\
\phi_{1}(t)=a_{1}\left(\beta_{2}-1\right) e^{b t}, \quad \phi_{2}(t)=a_{2}\left(\beta_{2}-1\right) e^{b t}, \quad \phi_{3}(t)=a_{3}\left(\beta_{2}-1\right) e^{b t}, \\
\varphi_{1}(t)=a_{3} e^{b t}, \quad \varphi_{2}(t)=a_{2} e^{b t}, \quad \varphi_{3}(t)=a_{1} e^{b t} .
\end{gathered}
$$


Therefore upper $\alpha$-cut of deterioration cost is given by

$$
\begin{aligned}
\text { D.C } & =\frac{C_{3}}{T}\{ \\
& a_{3} \frac{\left(\beta_{1}-1\right)\left(e^{b z}-1\right)}{b}-\frac{\alpha a_{3}\left(\beta_{1}-1\right)\left(e^{b z}-1\right)}{b}+\frac{a_{2} \alpha\left(\beta_{1}-1\right)\left(e^{b z}-1\right)}{b} \\
& +\frac{a_{3}\left(\beta_{2}-1\right)}{b}\left(e^{b \alpha_{1} t_{1}}-e^{b z}\right)-\frac{\alpha a_{3}\left(\beta_{2}-1\right)}{b}\left(e^{b \alpha_{1} t_{1}}-e^{b z}\right)+\frac{\alpha a_{2}\left(\beta_{2}-1\right)}{b}\left(e^{b \alpha_{1} t_{1}}-e^{b z}\right) \\
& \left.-\frac{a_{1}}{b}\left(e^{b t_{1}}-e^{b \alpha_{1} t_{1}}\right)-\frac{a_{1} \alpha}{b}\left(e^{b t_{1}}-e^{b \alpha_{1} t_{1}}\right)+\frac{a_{2} \alpha}{b}\left(e^{b t_{1}}-e^{b \alpha_{1} t_{1}}\right)\right\} .
\end{aligned}
$$

The lower $\alpha$-cut of deterioration cost is given by

$$
\begin{aligned}
& \text { D.C } C^{-}=\frac{C_{3}}{T}\left\{a_{1} \frac{\left(\beta_{1}-1\right)\left(e^{b z}-1\right)}{b}+\frac{\alpha a_{2}\left(\beta_{1}-1\right)\left(e^{b z}-1\right)}{b}-\frac{a_{1} \alpha\left(\beta_{1}-1\right)\left(e^{b z}-1\right)}{b}\right. \\
& +\frac{a_{1}\left(\beta_{2}-1\right)}{b}\left(e^{b \alpha_{1} t_{1}}-e^{b z}\right)+\frac{\alpha a_{2}\left(\beta_{2}-1\right)}{b}\left(e^{b \alpha_{1} t_{1}}-e^{b z}\right) \\
& -\frac{\alpha a_{1}\left(\beta_{2}-1\right)}{b}\left(e^{b \alpha_{1} t_{1}}-e^{b z}\right)-\frac{a_{3}}{b}\left(e^{b t_{1}}-e^{b \alpha_{1} t_{1}}\right)-\frac{a_{2} \alpha}{b}\left(e^{b t_{1}}-e^{b \alpha_{1} t_{1}}\right) \\
& \left.+\frac{a_{3} \alpha}{b}\left(e^{b t_{1}}-e^{b \alpha_{1} t_{1}}\right)\right\} \text {. }
\end{aligned}
$$

(3) The annual ordering cost $=C_{4} / T$.

Therefore total variable cost per unit time is a fuzzy quantity and is defined by

$$
\widetilde{\mathrm{TVC}^{2}}=\left\{\begin{array}{l}
\mathrm{TVC}^{+} \\
\mathrm{TVC}^{-}
\end{array}\right\}
$$

where

$$
\begin{aligned}
& \mathrm{TVC}^{+}=\sup \left\{x \in R: \mu_{\widetilde{\mathrm{TVC}}}(x) \geq \alpha\right\}, \\
& \mathrm{TVC}^{-}=\inf \left\{x \in R: \mu_{\widetilde{\mathrm{TVC}}}(x) \geq \alpha\right\} .
\end{aligned}
$$

The upper $\alpha$-cut of total variable cost per unit time is

$$
\begin{aligned}
\mathrm{TVC}^{+}= & \left(\frac{C_{1}}{T}\right)\left[A_{1}+B_{1} t_{1}+C_{1} t_{1}^{\beta+1}+D_{1} t_{1}^{\beta+2}+E_{1} t_{1}^{\beta+3}+F_{1} e^{b t_{1}} t_{1}+G_{1} e^{b t_{1}} t_{1}^{\beta+1}+H_{1} e^{b \alpha_{1} t_{1}}+I_{1} e^{b t_{1}}\right] \\
& +\frac{C_{2}}{T} \frac{\left\{a_{1}+\alpha\left(a_{2}-a_{1}\right)\right\}}{b}\left\{\frac{-e^{b \eta T}}{b}+\eta T e^{b t_{1}}+\frac{e^{b t_{1}}}{b}-t_{1} e^{b t_{1}}\right\}
\end{aligned}
$$




$$
\begin{aligned}
+\frac{C_{2}}{T} \frac{\left\{a_{1}+\alpha\left(a_{2}-a_{1}\right)\right\}\left(\beta_{1}-1\right)}{b^{2}}\left\{e^{b T} T-\frac{e^{b T}}{b}-\eta T e^{b T}+\frac{e^{b \eta T}}{b}\right\} \\
+\frac{C_{3}}{T}\left\{a_{3} \frac{\left(\beta_{1}-1\right)\left(e^{b z}-1\right)}{b}-\frac{\alpha a_{3}\left(\beta_{1}-1\right)\left(e^{b z}-1\right)}{b}+\frac{a_{2} \alpha\left(\beta_{1}-1\right)\left(e^{b z}-1\right)}{b}\right. \\
+\frac{a_{3}\left(\beta_{2}-1\right)}{b}\left(e^{b \alpha_{1} t_{1}}-e^{b z}\right)-\frac{\alpha a_{3}\left(\beta_{2}-1\right)}{b}\left(e^{b \alpha_{1} t_{1}}-e^{b z}\right) \\
+\frac{\alpha a_{2}\left(\beta_{2}-1\right)}{b}\left(e^{b \alpha_{1} t_{1}}-e^{b z}\right)-\frac{a_{1}}{b}\left(e^{b t_{1}}-e^{b \alpha_{1} t_{1}}\right)-\frac{a_{1} \alpha}{b}\left(e^{b t_{1}}-e^{b \alpha_{1} t_{1}}\right) \\
\left.+\frac{a_{2} \alpha}{b}\left(e^{b t_{1}}-e^{b \alpha_{1} t_{1}}\right)\right\}+\frac{C_{4}}{T} .
\end{aligned}
$$

The lower $\alpha$-cut of total variable cost per unit time is

$$
\begin{aligned}
\mathrm{TVC}^{-}= & \left(\frac{C_{1}}{T}\right)\left[A_{2}+B_{2} t_{1}+C_{2} t_{1}^{\beta+1}+D_{2} t_{1}^{\beta+2}+E_{2} t_{1}^{\beta+3}+F_{2} e^{b t_{1}} t_{1}+G_{2} e^{b t_{1}} t_{1}^{\beta+1}+H_{2} e^{b \alpha_{1} t_{1}}+I_{2} e^{b t_{1}}\right] \\
+ & \left(\frac{C_{2}}{T}\right) \frac{\left\{a_{3}-\alpha\left(a_{3}-a_{2}\right)\right\}}{b}\left\{\frac{-e^{b \eta T}}{b}+\eta T e^{b t_{1}}+\frac{e^{b t_{1}}}{b}-t_{1} e^{b t_{1}}\right\} \\
+ & \frac{C_{2}}{T} \frac{\left\{a_{3}-\alpha\left(a_{3}-a_{2}\right)\right\}\left(\beta_{1}-1\right)}{b^{2}}\left\{e^{b T} T-\frac{e^{b T}}{b}-\eta T e^{b T}+\frac{e^{b \eta T}}{b}\right\} \\
+ & \frac{C_{3}}{T}\left\{a_{1} \frac{\left(\beta_{1}-1\right)\left(e^{b z}-1\right)}{b}+\frac{\alpha a_{2}\left(\beta_{1}-1\right)\left(e^{b z}-1\right)}{b}-\frac{a_{1} \alpha\left(\beta_{1}-1\right)\left(e^{b z}-1\right)}{b}\right. \\
& +\frac{a_{1}\left(\beta_{2}-1\right)}{b}\left(e^{b \alpha_{1} t_{1}}-e^{b z}\right)+\frac{\alpha a_{2}\left(\beta_{2}-1\right)}{b}\left(e^{b \alpha_{1} t_{1}}-e^{b z}\right) \\
& -\frac{\alpha a_{1}\left(\beta_{2}-1\right)}{b}\left(e^{b \alpha_{1} t_{1}}-e^{b z}\right)-\frac{a_{3}}{b}\left(e^{b t_{1}}-e^{b \alpha_{1} t_{1}}\right)-\frac{a_{2} \alpha}{b}\left(e^{b t_{1}}-e^{b \alpha_{1} t_{1}}\right) \\
& \left.+\frac{a_{3} \alpha}{b}\left(e^{b t_{1}}-e^{b \alpha_{1} t_{1}}\right)\right\}+\left(\frac{C_{4}}{T}\right) .
\end{aligned}
$$

The objective in this paper is to find an optimal cycle time to minimize the total variable cost per unit time.

Therefore this model mathematically can be written as

$$
\begin{array}{ll}
\text { Minimize } & \left\{\mathrm{TVC}^{+}, \mathrm{TVC}^{-}\right\} \\
\text {Subject to } & 0 \leq \alpha \leq 1 .
\end{array}
$$


Therefore the problem is a multiobjective optimization problem. To convert it as a singleobjective optimization problem we use global criteria (GC) method.

Then the above problem reduces to

$$
\begin{array}{ll}
\text { Minimize } & \text { GC } \\
\text { Subject to } & 0 \leq \alpha \leq 1 .
\end{array}
$$

\section{Global Criteria Method}

The model presented by (4.26) is a multiobjective model which is solved by Global Criteria (GC) Method with the help of Generalized Reduced Gradient Technique.

The Multiobjective Nonlinear Integer Programming (MONLIP) problems are solved by Global Criteria method converting it to a single-objective optimization problem. The solution procedure is as follows.

Step 1. Solve the multiobjective programming problem (4.26) as a single-objective problem using only one objective at a time ignoring others.

Step 2. From the results of Step 1, determine the ideal objective vector, say $\left(\mathrm{TVC}^{+\mathrm{min}}\right.$, $\mathrm{TVC}^{-\mathrm{min}}$ ) and the corresponding values of $\left(\mathrm{TVC}^{+\max }, \mathrm{TVC}^{-\max }\right)$. Here, the ideal objective vector is used as a reference point. The problem is then to solve the following auxiliary problem:

$$
\operatorname{Min}(\mathrm{GC})=\operatorname{Minimize}\left\{\left(\frac{\mathrm{TVC}^{+}-\mathrm{TVC}^{+\min }}{\mathrm{TVC}^{+\max }-\mathrm{TVC}^{+\min }}\right)^{Q}+\left(\frac{\mathrm{TVC}^{-}-\mathrm{TVC}^{-\min }}{\mathrm{TVC}^{-\max }-\mathrm{TVC}^{-\mathrm{min}}}\right)^{Q}\right\}^{1 / Q}
$$

where $1 \leq Q<\infty$. This method is also sometimes called Compromise Programming.

\section{Numerical Example}

We now consider a numerical example showing the utility of the model from practical point of view. According to the developed solution procedure of the proposed inventory system, the optimal solution has been obtained with the help of well-known generalized reduced gradient method (GRG). To illustrate the developed model, an example with the following data has been considered.

Let $a_{1}=100$ units $/$ month, $a_{2}=200$ units $/$ month, $a_{3}=100$ units $/$ month, $C_{1}=\$ .1$ per unit, $C_{2}=\$ .5$ per unit, $C_{3}=\$ .2$ per unit, $C_{4}=\$ 100$ per order, $b=.2, \alpha=.25, \beta=1.9$, $\beta_{1}=0.9, \beta_{2}=1.9, \Upsilon=.001 T=7 \mathrm{hrs}$.

Substituting the above parameters, Global Criteria (GC) is obtained as

$$
\mathrm{GC}=0.0926
$$

The compromise solutions are $\mathrm{TVC}^{+}=\$ 548.62, \mathrm{TVC}^{-}=\$ 503.74$. $^{-}$ 


\section{Conclusion}

In this paper the multiobjective problem is solved by Global Criteria method. In reality, in different systems, there are some parameters which are imprecise in nature. The present paper proposes a solution procedure to develop an EPQ inventory model with variable production rate and fuzzy demand. In most of the real life problem demand of a newly launched product is not known in advance. This justifies the introduction of fuzzy demand. The technique for multiobjective optimization may be applied to the areas like environmental analysis, transportation, and so forth. In this paper we have taken two rates of production but this can be extended to $n$ number of production rates $p_{i}$ during the time when the inventory level goes from $(i-1) Q$ to $i Q(i=1,2, \ldots, n)$, where $Q$ is prefixed level.

\section{Appendices}

\section{A. The Upper $\alpha$-Cut of Fuzzy Stock Holding Cost}

The upper $\alpha$-cut of fuzzy stock holding cost is given by:

$$
\begin{aligned}
& H C^{+}=\left(\frac{C_{1}}{T}\right)\left[\int_{0}^{z} I_{1}^{+}(t) d t+\int_{z}^{\alpha_{1} t_{1}} I_{2}^{+}(t) d t+\int_{\alpha_{1} t_{1}}^{t_{1}} I_{3}^{+}(t) d t\right] \\
& =\left(\frac{C_{1}}{T}\right)\left[\frac{\left(\beta_{1}-1\right)\left\{a_{3}-\alpha\left(a_{3}-a_{2}\right)\right\}}{b}\left(\frac{e^{b z}}{b}-z-\frac{1}{b}\right)-\frac{\gamma \beta\left(\beta_{1}-1\right) a_{3} z^{\beta+2}}{(1+\beta)(2+\beta)}\right. \\
& +P\left\{\alpha_{1} t_{1}+\gamma \alpha_{1} z^{\beta} t_{1}-\frac{\gamma \alpha_{1}^{\beta+1} t_{1}^{\beta+1}}{(\beta+1)}-z-\gamma z^{\beta+1}+\frac{\gamma z^{\beta+1}}{\beta+1}\right\} \\
& +\frac{\left(\beta_{2}-1\right)}{b}\left\{a_{3}-\alpha\left(a_{3}-a_{2}\right)\right\}\left\{\frac{e^{b \alpha_{1} t_{1}}}{b}-\alpha_{1} t_{1} e^{b z}-\frac{e^{b z}}{b}+z e^{b z}\right\} \\
& +\frac{\gamma a_{3}\left(\beta_{2}-1\right) \beta \alpha_{1}^{\beta+1} t_{1}^{\beta+1}}{b(1+\beta)}-\frac{\gamma a_{3}\left(\beta_{2}-1\right) \beta z^{\beta+1}}{b(1+\beta)} \\
& -\frac{r a_{3}\left(\beta_{2}-1\right)}{b}\left[\left\{\left(\alpha_{1} t_{1}\right)^{\beta+1}-z^{\beta+1}\right\}+\frac{b}{2}\left\{\left(\alpha_{1} t_{1}\right)^{\beta+2}-z^{\beta+2}\right\}\right. \\
& \left.+\frac{b^{2}}{6}\left\{\left(\alpha_{1} t_{1}\right)^{\beta+3}-z^{\beta+3}\right\}\right]+\frac{\gamma a_{3}\left(\beta_{2}-1\right) e^{b z}}{b(1+\beta)}\left\{\left(\alpha_{1} t_{1}\right)^{\beta+1}-z^{\beta+1}\right\} \\
& -\frac{\gamma\left\{a_{3}-\alpha\left(a_{3}-a_{2}\right)\right\}\left(\beta_{2}-1\right) \beta \alpha_{1}^{\beta+1} t_{1}^{\beta+1}}{b(\beta+1)}+\frac{\gamma\left\{a_{3}-\alpha\left(a_{3}-a_{2}\right)\right\}\left(\beta_{2}-1\right) \beta z^{\beta+1}}{b(1+\beta)} \\
& +\frac{r\left\{a_{3}-\alpha\left(a_{3}-a_{2}\right)\right\}\left(\beta_{2}-1\right)}{b}\left[\left\{\left(\alpha_{1} t_{1}\right)^{\beta+1}-z^{\beta+1}\right\}+\frac{b}{2}\left\{\left(\alpha_{1} t_{1}\right)^{\beta+2}-z^{\beta+2}\right\}\right. \\
& \left.+\frac{b^{2}}{6}\left\{\left(\alpha_{1} t_{1}\right)^{\beta+3}-z^{\beta+3}\right\}\right]
\end{aligned}
$$




$$
\begin{aligned}
& -\frac{\gamma\left\{a_{3}-\alpha\left(a_{3}-a_{2}\right)\right\}\left(\beta_{2}-1\right)}{b(\beta+1)}\left\{\left(\alpha_{1} t_{1}\right)^{\beta+1}-z^{\beta+1}\right\} \\
& -\frac{\gamma\left\{a_{3}+\alpha\left(a_{3}-a_{2}\right)\right\}\left(\beta_{2}-1\right) z^{\beta} e^{b z}\left(\alpha_{1} t_{1}-z\right)}{b} \\
& +\frac{\gamma\left\{a_{3}-\alpha\left(a_{3}-a_{2}\right)\right\}\left(\beta_{2}-1\right) z^{\beta}\left(\alpha_{1} t_{1}-z\right)}{b} \\
& +\frac{\gamma\left\{a_{3}-\alpha\left(a_{3}-a_{2}\right)\right\} \beta\left(\beta_{2}-1\right)}{(1+\beta)(2+\beta)}\left\{(\beta+2) z^{\beta+1} \alpha_{1} t_{1}-\alpha_{1}^{\beta+2} t_{1}^{\beta+2}-z^{\beta+2}(\beta+2)+z^{\beta+2}\right\} \\
& +\frac{\left\{a_{1}+\alpha\left(a_{2}-a_{1}\right)\right\}}{b}\left\{e^{b t_{1}} t_{1}-\frac{e^{b t_{1}}}{b}-\alpha_{1} t_{1} e^{b t_{1}}+\frac{e^{b \alpha_{1} t_{1}}}{b}\right\}-\frac{a_{1} \gamma e^{b t_{1}}\left(t_{1}^{\beta+1}-\alpha_{1}^{\beta+1} t_{1}^{\beta+1}\right)}{b(\beta+1)} \\
& -\frac{a_{1} \gamma \beta}{b(1+\beta)}\left\{t_{1}^{\beta+1}-\alpha_{1}^{\beta+1} t_{1}^{\beta+1}\right\}+\frac{a_{1} \gamma}{b}\left\{\left(t_{1}^{\beta+1}-\alpha_{1}^{\beta+1} t_{1}^{\beta+1}\right)+\frac{b}{2}\left(t_{1}^{\beta+2}-\alpha_{1}^{\beta+2} t_{1}^{\beta+2}\right)\right. \\
& \left.+\frac{b^{2}}{6}\left(t_{1}^{\beta+3}-\alpha_{1}^{\beta+3} t_{1}^{\beta+3}\right)\right\} \\
& -\frac{\left\{a_{1}+\alpha\left(a_{2}-a_{12}\right)\right\} \gamma}{b}\left\{t_{1}^{\beta+1} e^{b t_{1}}-t_{1}^{\beta+1}-\alpha_{1} t_{1}^{\beta+1} e^{b t_{1}}+\alpha_{1} t_{1}^{\beta+1}\right\} \\
& +\frac{\beta \gamma\left\{a_{1}+\alpha\left(a_{2}-a_{12}\right)\right\}}{b(1+\beta)}\left(t_{1}^{\beta+1}-\alpha_{1}^{\beta+1} t_{1}^{\beta+1}\right) \\
& -\frac{\gamma\left\{a_{1}+\alpha\left(a_{2}-a_{1}\right)\right\}}{b}\left\{\left(t_{1}^{\beta+1}-\alpha_{1}^{\beta+1} t_{1}^{\beta+1}\right)+\frac{b}{2}\left(t_{1}^{\beta+2}-\alpha_{1}^{\beta+2} t_{1}^{\beta+2}\right)\right. \\
& \left.+\frac{b^{2}}{6}\left(t_{1}^{\beta+3}-\alpha_{1}^{\beta+3} t_{1}^{\beta+3}\right)\right\} \\
& +\frac{\gamma\left\{a_{1}+\alpha\left(a_{2}-a_{1}\right)\right\}\left(t_{1}^{\beta+1}-\alpha_{1}^{\beta+1} t_{1}^{\beta+1}\right)}{b(1+\beta)} \\
& \left.-\left\{a_{1}+\alpha\left(a_{2}-a_{1}\right)\right\} \frac{\gamma \beta}{(1+\beta)}\left\{t_{1}^{\beta+2}-\frac{t_{1}^{\beta+2}}{(\beta+2)}-\alpha_{1} t_{1}^{\beta+2}+\frac{\alpha_{1}^{\beta+2} t_{1}^{\beta+2}}{(\beta+2)}\right\}\right] \\
& H C^{+}=\left(\frac{C_{1}}{T}\right)\left[\int_{0}^{z} I_{1}^{+}(t) d t+\int_{z}^{\alpha_{1} t_{1}} I_{2}^{+}(t) d t+\int_{\alpha_{1} t_{1}}^{t_{1}} I_{3}^{+}(t) d t\right] \\
& =\left(\frac{C_{1}}{T}\right)\left[A_{1}+B_{1} t_{1}+C_{1} t_{1}^{\beta+1}+D_{1} t_{1}^{\beta+2}+E_{1} t_{1}^{\beta+3}+F_{1} e^{b t_{1}} t_{1}+G_{1} e^{b t_{1}} t_{1}^{\beta+1}+H_{1} e^{b \alpha_{1} t_{1}}+I_{1} e^{b t_{1}}\right],
\end{aligned}
$$


where

$$
\begin{aligned}
& A_{1}=\frac{\left(\beta_{1}-1\right)\left\{a_{3}-\alpha\left(a_{3}-a_{2}\right)\right\}}{b}\left(\frac{e^{b z}}{b}-z-\frac{1}{b}\right)-\frac{\gamma \beta\left(\beta_{1}-1\right) a_{3} z^{\beta+2}}{(1+\beta)(2+\beta)}-P z-P \gamma z^{\beta+1}+P \frac{\gamma z^{\beta+1}}{\beta+1} \\
& +\frac{\left(\beta_{2}-1\right)}{b}\left\{a_{3}-\alpha\left(a_{3}-a_{2}\right)\right\}\left\{-\frac{e^{b z}}{b}+z e^{b z}\right\}-\frac{\gamma a_{3}\left(\beta_{2}-1\right) \beta z^{\beta+1}}{b(1+\beta)} \\
& +\frac{\gamma a_{3}\left(\beta_{2}-1\right)}{b}\left\{z^{\beta+1}+\frac{b}{2} z^{\beta+2}+\frac{b^{2}}{6} z^{\beta+3}\right\}-\frac{\gamma a_{3}\left(\beta_{2}-1\right) e^{b z}}{b(1+\beta)} z^{\beta+1} \\
& +\frac{\gamma\left\{a_{3}-\alpha\left(a_{3}-a_{2}\right)\right\}\left(\beta_{2}-1\right) \beta z^{\beta+1}}{b(1+\beta)}-\frac{\gamma\left\{a_{3}-\alpha\left(a_{3}-a_{2}\right)\right\}\left(\beta_{2}-1\right)}{b}\left\{z^{\beta+1}+\frac{b}{2} z^{\beta+2}+\frac{b^{2}}{6} z^{\beta+3}\right\} \\
& +\frac{\gamma\left\{a_{3}-\alpha\left(a_{3}-a_{2}\right)\right\}\left(\beta_{2}-1\right)}{b(\beta+1)} z^{\beta+1}+\frac{\gamma\left\{a_{3}-\alpha\left(a_{3}-a_{2}\right)\right\}\left(\beta_{2}-1\right) z^{\beta+1} e^{b z}}{b} \\
& -\frac{\left\{a_{3}-\alpha\left(a_{3}-a_{2}\right)\right\} \gamma\left(\beta_{2}-1\right) z^{\beta+1}}{b}-\frac{\gamma\left\{a_{3}-\alpha\left(a_{3}-a_{2}\right)\right\} \beta\left(\beta_{2}-1\right)}{(2+\beta)} z^{\beta+2}, \\
& B_{1}=\operatorname{Pr}+\operatorname{Pr} \alpha_{1} z^{\beta}-\frac{\left(\beta_{2}-1\right)}{b}\left\{a_{3}-\alpha\left(a_{3}-a_{2}\right)\right\} \alpha_{1} e^{b z}-\frac{\gamma\left\{a_{3}-\alpha\left(a_{3}-a_{2}\right)\right\}\left(\beta_{2}-1\right) z^{\beta} e^{b z} \alpha_{1}}{b} \\
& +\frac{\gamma\left\{a_{3}-\alpha\left(a_{3}-a_{2}\right)\right\}\left(\beta_{2}-1\right) z^{\beta} \alpha_{1}}{b}+\frac{\gamma\left\{a_{3}-\alpha\left(a_{3}-a_{2}\right)\right\} \beta\left(\beta_{2}-1\right)}{(1+\beta)} z^{\beta+1} \alpha_{1}, \\
& C_{1}=\left[\frac{-P \gamma \alpha_{1}^{\beta+1}}{(\beta+1)}+\frac{\gamma a_{3}\left(\beta_{2}-1\right) \beta \alpha_{1}^{\beta+1}}{b(1+\beta)}-\frac{\gamma a_{3}\left(\beta_{2}-1\right)}{b} \alpha_{1}^{\beta+1}+\frac{\gamma a_{3}\left(\beta_{2}-1\right) e^{b z}}{b(1+\beta)} \alpha_{1}^{\beta+1}\right. \\
& -\frac{\gamma\left\{a_{3}-\alpha\left(a_{3}-a_{2}\right)\right\}\left(\beta_{2}-1\right) \beta \alpha_{1}^{\beta+1}}{b(\beta+1)}+\frac{\gamma\left\{a_{3}-\alpha\left(a_{3}-a_{2}\right)\right\}\left(\beta_{2}-1\right)}{b} \alpha_{1}^{\beta+1} \\
& -\frac{\gamma\left\{a_{3}-\alpha\left(a_{3}-a_{2}\right)\right\}\left(\beta_{2}-1\right)}{b(\beta+1)} \alpha_{1}^{\beta+1}-\frac{a_{1} \gamma e^{b t_{1}}\left(1-\alpha_{1}^{\beta+1}\right)}{b(\beta+1)}-\frac{a_{1} \gamma \beta}{b(1+\beta)}\left(1-\alpha_{1}^{\beta+1}\right) \\
& +\frac{a_{1} \gamma}{b}\left(1-\alpha_{1}^{\beta+1}\right)-\frac{\left\{a_{1}+\alpha\left(a_{2}-a_{1}\right)\right\} \gamma}{b}\left(\alpha_{1}-1\right)+\frac{\beta \gamma\left\{a_{1}+\alpha\left(a_{2}-a_{1}\right)\right\}}{b(1+\beta)}\left(1-\alpha_{1}^{\beta+1}\right) \\
& \left.-\frac{\gamma\left\{a_{1}+\alpha\left(a_{2}-a_{1}\right)\right\}}{b}\left(1-\alpha_{1}^{\beta+1}\right)+\frac{\gamma\left\{a_{1}+\alpha\left(a_{2}-a_{1}\right)\right\}\left(1-\alpha_{1}^{\beta+1}\right)}{b(1+\beta)}\right],
\end{aligned}
$$


Advances in Operations Research

17

$$
\begin{aligned}
D_{1}= & -\frac{\gamma a_{3}\left(\beta_{2}-1\right) \alpha_{1}^{\beta+2}}{2}+\frac{\gamma\left\{a_{3}-\alpha\left(a_{3}-a_{2}\right)\right\}\left(\beta_{2}-1\right) \alpha_{1}^{\beta+2}}{2}-\frac{\left\{a_{3}-\alpha\left(a_{3}-a_{2}\right)\right\} \gamma \beta\left(\beta_{2}-1\right)}{(1+\beta)(2+\beta)} \alpha_{1}^{\beta+2} \\
& -\frac{a_{1} \gamma\left(1-\alpha_{1}^{\beta+2}\right)}{2}-\frac{\gamma\left\{a_{1}+\alpha\left(a_{2}-a_{1}\right)\right\}\left(1-\alpha_{1}^{\beta+2}\right)}{2}-\left\{a_{1}+\alpha\left(a_{2}-a_{1}\right)\right\} \frac{\gamma \beta}{(2+\beta)} \\
& +\left\{a_{1}+\alpha\left(a_{2}-a_{1}\right)\right\} \frac{\gamma \alpha_{1} \beta}{(1+\beta)}-\frac{\alpha_{1}^{\beta+2} \gamma \beta}{(\beta+1)(\beta+2)}\left\{a_{1}+\alpha\left(a_{2}-a_{1}\right)\right\}, \\
E_{1}= & -\frac{\gamma a_{3}\left(\beta_{2}-1\right) b \alpha_{1}^{\beta+3}}{6}+\frac{\left\{a_{3}-\alpha\left(a_{3}-a_{2}\right)\right\}\left(\beta_{2}-1\right) \gamma b \alpha_{1}^{\beta+3}}{6}+\frac{a_{1} \gamma b}{6}\left(1-\alpha_{1}^{\beta+3}\right) \\
& -\frac{\gamma b\left\{a_{1}+\alpha\left(a_{2}-a_{1}\right)\right\}\left(1-\alpha_{1}^{\beta+3}\right)}{6}, \\
F_{1}= & \frac{\left\{a_{1}+\alpha\left(a_{2}-a_{1}\right)\right\}}{b}\left(1-\alpha_{1}\right), \\
G_{1}= & \frac{a_{1} \gamma\left(\alpha_{1}^{\beta+1}-1\right)}{b(\beta+1)}+\frac{\left\{a_{1}+\alpha\left(a_{2}-a_{1}\right)\right\} \gamma}{b}\left(\alpha_{1}-1\right), \\
I_{1}= & -\frac{\left\{a_{1}+\alpha\left(a_{2}-a_{1}\right)\right\}}{b^{2}} . \\
H_{1}= & \frac{\left\{a_{1}+\alpha\left(a_{2}-a_{1}\right)\right\}}{b^{2}}+\frac{\left(\beta_{2}-1\right)}{b^{2}}\left\{a_{3}-\alpha\left(a_{3}-a_{2}\right)\right\}, \\
&
\end{aligned}
$$

B. The Lower $\boldsymbol{\alpha}$-Cut of Fuzzy Stock Holding Cost

The lower $\alpha$-cut of fuzzy stock holding cost is given by:

$$
\begin{aligned}
H C^{-}=\left(\frac{C_{1}}{T}\right)\left[\int_{0}^{z} I_{1}^{-}(t) d t+\int_{z}^{\alpha_{1} t_{1}} I_{2}^{-}(t) d t+\int_{\alpha_{1} t_{1}}^{t_{1}} I_{3}^{-}(t) d t\right] \\
=\left(\frac{C_{1}}{T}\right)\left[\frac{\left(\beta_{1}-1\right)\left\{a_{1}+\alpha\left(a_{2}-a_{1}\right)\right\}}{b}\left(\frac{e^{b z}}{b}-z-\frac{1}{b}\right)-\frac{\gamma \beta\left(\beta_{1}-1\right) a_{1} z^{\beta+2}}{(1+\beta)(2+\beta)}\right. \\
+P\left\{\alpha_{1} t_{1}+\gamma \alpha_{1} z^{\beta} t_{1}-\frac{\gamma \alpha_{1}^{\beta+1} t_{1}^{\beta+1}}{(\beta+1)}-z-\gamma z^{\beta+1}+\frac{\gamma z^{\beta+1}}{\beta+1}\right\} \\
+\frac{\left(\beta_{2}-1\right)}{b}\left\{a_{1}+\alpha\left(a_{2}-a_{1}\right)\right\}\left\{\frac{e^{b \alpha_{1} t_{1}}}{b}-\alpha_{1} t_{1} e^{b z}-\frac{e^{b z}}{b}+z e^{b z}\right\}
\end{aligned}
$$


18

Advances in Operations Research

$$
\begin{aligned}
& +\frac{\gamma a_{1}\left(\beta_{2}-1\right) \beta \alpha_{1}^{\beta+1} t_{1}^{\beta+1}}{b(1+\beta)}-\frac{\gamma a_{1}\left(\beta_{2}-1\right) \beta z^{\beta+1}}{b(1+\beta)} \\
& -\frac{\gamma a_{1}\left(\beta_{2}-1\right)}{b}\left[\left\{\left(\alpha_{1} t_{1}\right)^{\beta+1}-z^{\beta+1}\right\}+\frac{b}{2}\left\{\left(\alpha_{1} t_{1}\right)^{\beta+2}-z^{\beta+2}\right\}\right. \\
& \left.+\frac{b^{2}}{6}\left\{\left(\alpha_{1} t_{1}\right)^{\beta+3}-z^{\beta+3}\right\}\right]+\frac{\gamma a_{1}\left(\beta_{2}-1\right) e^{b z}}{b(1+\beta)}\left\{\left(\alpha_{1} t_{1}\right)^{\beta+1}-z^{\beta+1}\right\} \\
& -\frac{\gamma\left\{a_{1}+\alpha\left(a_{2}-a_{1}\right)\right\}\left(\beta_{2}-1\right) \beta\left(\alpha_{1}^{\beta+1} t_{1}^{\beta+1}-z^{\beta+1}\right)}{b(\beta+1)} \\
& +\frac{\gamma\left\{a_{1}+\alpha\left(a_{2}-a_{1}\right)\right\}\left(\beta_{2}-1\right)}{b}\left[\left\{\left(\alpha_{1} t_{1}\right)^{\beta+1}-z^{\beta+1}\right\}+\frac{b}{2}\left\{\left(\alpha_{1} t_{1}\right)^{\beta+2}-z^{\beta+2}\right\}\right. \\
& \left.+\frac{b^{2}}{6}\left\{\left(\alpha_{1} t_{1}\right)^{\beta+3}-z^{\beta+3}\right\}\right] \\
& -\frac{\gamma\left\{a_{1}+\alpha\left(a_{2}-a_{1}\right)\right\}\left(\beta_{2}-1\right)}{b(\beta+1)}\left\{\left(\alpha_{1} t_{1}\right)^{\beta+1}-z^{\beta+1}\right\} \\
& +\gamma\left\{a_{1}+\alpha\left(a_{2}-a_{1}\right)\right\} \frac{\left(\beta_{2}-1\right) z^{\beta}\left(1-e^{b z}\right)\left(\alpha_{1} t_{1}-z\right)}{b} \\
& +\frac{\left\{a_{1}+\alpha\left(a_{2}-a_{1}\right)\right\} \beta\left(\beta_{2}-1\right)}{(1+\beta)(2+\beta)}\left\{(\beta+2) z^{\beta+1} \alpha_{1} t_{1}-\alpha_{1}^{\beta+2} t_{1}^{\beta+2}-z^{\beta+2}(\beta+2)+z^{\beta+2}\right\} \\
& +\frac{\left\{a_{3}-\alpha\left(a_{3}-a_{2}\right)\right\}}{b}\left\{e^{b t_{1}} t_{1}-\frac{e^{b t_{1}}}{b}-\alpha_{1} t_{1} e^{b t_{1}}+\frac{e^{b \alpha_{1} t_{1}}}{b}\right\} \\
& -\frac{a_{3} \gamma e^{b t_{1}}\left(t_{1}^{\beta+1}-\alpha_{1}^{\beta+1} t_{1}^{\beta+1}\right)}{b(\beta+1)}-\frac{a_{3} \gamma \beta}{b(1+\beta)}\left\{t_{1}^{\beta+1}-\alpha_{1}^{\beta+1} t_{1}^{\beta+1}\right\} \\
& +\frac{a_{3} \gamma}{b}\left\{\left(t_{1}^{\beta+1}-\alpha_{1}^{\beta+1} t_{1}^{\beta+1}\right)+\frac{b}{2}\left(t_{1}^{\beta+2}-\alpha_{1}^{\beta+2} t_{1}^{\beta+2}\right)+\frac{b^{2}}{6}\left(t_{1}^{\beta+3}-\alpha_{1}^{\beta+3} t_{1}^{\beta+3}\right)\right\} \\
& -\frac{\left\{a_{3}-\alpha\left(a_{3}-a_{2}\right)\right\} \gamma}{b}\left\{t_{1}^{\beta+1} e^{b t_{1}}-t_{1}^{\beta+1}-\alpha_{1} t_{1}^{\beta+1} e^{b t_{1}}+\alpha_{1} t_{1}^{\beta+1}\right\} \\
& +\frac{\beta \gamma\left\{a_{3}-\alpha\left(a_{3}-a_{2}\right)\right\}}{\mathrm{b}(1+\beta)}\left(t_{1}^{\beta+1}-\alpha_{1}^{\beta+1} t_{1}^{\beta+1}\right) \\
& -\frac{\gamma\left\{a_{3}-\alpha\left(a_{3}-a_{2}\right)\right\}}{b}\left\{\left(t_{1}^{\beta+1}-\alpha_{1}^{\beta+1} t_{1}^{\beta+1}\right)+\frac{b}{2}\left(t_{1}^{\beta+2}-\alpha_{1}^{\beta+2} t_{1}^{\beta+2}\right)\right. \\
& \left.+\frac{b^{2}}{6}\left(t_{1}^{\beta+3}-\alpha_{1}^{\beta+3} t_{1}^{\beta+3}\right)\right\}
\end{aligned}
$$


Advances in Operations Research

$$
\begin{gathered}
+\frac{\gamma\left\{a_{3}-\alpha\left(a_{3}-a_{2}\right)\right\}\left(t_{1}^{\beta+1}-\alpha_{1}^{\beta+1} t_{1}^{\beta+1}\right)}{b(1+\beta)} \\
\left.-\left\{a_{3}-\alpha\left(a_{3}-a_{2}\right)\right\} \frac{\gamma \beta}{(1+\beta)}\left\{t_{1}^{\beta+2}-\frac{t_{1}^{\beta+2}}{(\beta+2)}-\alpha_{1} t_{1}^{\beta+2}+\frac{\alpha_{1}^{\beta+2} t_{1}^{\beta+2}}{(\beta+2)}\right\}\right] \\
=\left(\frac{C_{1}}{T}\right)\left[A_{2}+B_{2} t_{1}+C_{2} t_{1}^{\beta+1}+D_{2} t_{1}^{\beta+2}+E_{2} t_{1}^{\beta+3}+F_{2} e^{b t_{1}} t_{1}+G_{2} e^{b t_{1}} t_{1}^{\beta+1}+H_{2} e^{b \alpha_{1} t_{1}}+I_{2} e^{b t_{1}}\right],
\end{gathered}
$$

where

$$
\begin{aligned}
& A_{2}=\frac{\left(\beta_{1}-1\right)\left\{a_{1}+\alpha\left(a_{2}-a_{1}\right)\right\}}{b}\left(\frac{e^{b z}}{b}-z-\frac{1}{b}\right)-\frac{\gamma \beta\left(\beta_{1}-1\right) a_{1} z^{\beta+2}}{(1+\beta)(2+\beta)}-P z-P \gamma z^{\beta+1} \\
& +P \frac{\gamma z^{\beta+1}}{\beta+1}+\frac{\left(\beta_{2}-1\right)}{b}\left\{a_{1}+\alpha\left(a_{2}-a_{1}\right)\right\}\left\{-\frac{e^{b z}}{b}+z e^{b z}\right\} \\
& -\frac{\gamma a_{1}\left(\beta_{2}-1\right) \beta z^{\beta+1}}{b(1+\beta)}+\frac{\gamma a_{1}\left(\beta_{2}-1\right)}{b}\left\{z^{\beta+1}+\frac{b}{2} z^{\beta+2}+\frac{b^{2}}{6} z^{\beta+3}\right\} \\
& -\frac{\gamma a_{1}\left(\beta_{2}-1\right) e^{b z}}{b(1+\beta)} z^{\beta+1}+\frac{\gamma\left\{a_{1}+\alpha\left(a_{2}-a_{1}\right)\right\}\left(\beta_{2}-1\right) \beta z^{\beta+1}}{b(1+\beta)} \\
& -\frac{\left\{a_{1}+\alpha\left(a_{2}-a_{1}\right)\right\} \gamma\left(\beta_{2}-1\right)}{b}\left\{z^{\beta+1}+\frac{b}{2} z^{\beta+2}+\frac{b^{2}}{6} z^{\beta+3}\right\} \\
& +\frac{\left\{a_{1}+\alpha\left(a_{2}-a_{1}\right)\right\}\left(\beta_{2}-1\right) \gamma}{b(\beta+1)} z^{\beta+1}+\frac{\left\{a_{1}+\alpha\left(a_{2}-a_{1}\right)\right\} \gamma\left(\beta_{2}-1\right) z^{\beta+1} e^{b z}}{b} \\
& -\frac{\gamma\left\{a_{1}+\alpha\left(a_{2}-a_{1}\right)\right\}\left(\beta_{2}-1\right) z^{\beta+1}}{b}-\frac{\gamma\left\{a_{3}-\alpha\left(a_{3}-a_{2}\right)\right\} \beta\left(\beta_{2}-1\right)}{(2+\beta)} z^{\beta+2}, \\
& B_{2}=\operatorname{Pr}+\operatorname{Pr} \alpha_{1} z^{\beta}-\frac{\left(\beta_{2}-1\right)}{b}\left\{a_{1}+\alpha\left(a_{2}-a_{1}\right)\right\} \alpha_{1} e^{b z} \\
& -\frac{\left\{a_{1}+\alpha\left(a_{2}-a_{1}\right)\right\}\left(\beta_{2}-1\right) \gamma z^{\beta} e^{b z} \alpha_{1}}{b}+\frac{\gamma\left\{a_{1}+\alpha\left(a_{2}-a_{1}\right)\right\}\left(\beta_{2}-1\right) z^{\beta} \alpha_{1}}{b} \\
& +\frac{\gamma\left\{a_{1}+\alpha\left(a_{2}-a_{1}\right)\right\} \beta\left(\beta_{2}-1\right)}{(1+\beta)} z^{\beta+1} \alpha_{1} \\
& C_{2}=\left[\frac{-P \gamma \alpha_{1}^{\beta+1}}{(\beta+1)}+\frac{\gamma a_{1}\left(\beta_{2}-1\right) \beta \alpha_{1}^{\beta+1}}{b(1+\beta)}-\frac{\gamma a_{1}\left(\beta_{2}-1\right)}{b} \alpha_{1}^{\beta+1}\right. \\
& +\frac{\alpha a_{1}\left(\beta_{2}-1\right) e^{b z}}{b(1+\beta)} \alpha_{1}^{\beta+1}-\frac{\gamma\left\{a_{1}+\alpha\left(a_{2}-a_{1}\right)\right\}\left(\beta_{2}-1\right) \beta \alpha_{1}^{\beta+1}}{b(\beta+1)}
\end{aligned}
$$




$$
\begin{aligned}
& +\frac{\gamma\left\{a_{1}+\alpha\left(a_{2}-a_{1}\right)\right\}\left(\beta_{2}-1\right)}{b} \alpha_{1}^{\beta+1}-\frac{\gamma\left\{a_{1}+\alpha\left(a_{2}-a_{1}\right)\right\}\left(\beta_{2}-1\right)}{b(\beta+1)} \alpha_{1}{ }^{\beta+1} \\
& -\frac{a_{3} \gamma e^{b t_{1}}\left(1-\alpha_{1}^{\beta+1}\right)}{b(\beta+1)}-\frac{a_{3} \gamma \beta}{b(1+\beta)}\left(1-\alpha_{1}^{\beta+1}\right)+\frac{a_{3} \gamma}{b}\left(1-\alpha_{1}^{\beta+1}\right) \\
& -\frac{\left\{a_{3}-\alpha\left(a_{3}-a_{2}\right)\right\} \gamma}{b}\left(\alpha_{1}-1\right)+\frac{\beta \gamma\left\{a_{3}-\alpha\left(a_{3}-a_{2}\right)\right\}}{b(1+\beta)}\left(1-\alpha_{1}^{\beta+1}\right) \\
& \left.-\frac{\gamma\left\{a_{3}-\alpha\left(a_{3}-a_{2}\right)\right\}}{b}\left(1-\alpha_{1}^{\beta+1}\right)+\frac{\gamma\left\{a_{3}-\alpha\left(a_{3}-a_{2}\right)\right\}\left(1-\alpha_{1}^{\beta+1}\right)}{b(1+\beta)}\right], \\
& D_{2}=-\frac{\gamma a_{1}\left(\beta_{2}-1\right) \alpha_{1}^{\beta+2}}{2}+\frac{\gamma\left\{a_{1}+\alpha\left(a_{2}-a_{1}\right)\right\}\left(\beta_{2}-1\right) \alpha_{1}^{\beta+2}}{2} \\
& -\frac{\left\{a_{1}+\alpha\left(a_{2}-a_{1}\right)\right\} \beta \gamma\left(\beta_{2}-1\right)}{(1+\beta)(2+\beta)} \alpha_{1}^{\beta+2}-\frac{a_{3} \gamma\left(1-\alpha_{1}^{\beta+2}\right)}{2}-\frac{\gamma\left\{a_{3}-\alpha\left(a_{3}-a_{2}\right)\right\}\left(1-\alpha_{1}^{\beta+2}\right)}{2} \\
& -\left\{a_{3}-\alpha\left(a_{3}-a_{2}\right)\right\} \frac{\gamma \beta}{(2+\beta)}+\left\{a_{3}-\alpha\left(a_{3}-a_{2}\right)\right\} \frac{\gamma \alpha_{1} \beta}{(1+\beta)} \\
& -\frac{\alpha_{1}^{\beta+2} \gamma \beta}{(\beta+1)(\beta+2)}\left\{a_{3}-\alpha\left(a_{3}-a_{2}\right)\right\} \text {, } \\
& E_{2}=-\frac{\gamma a_{1}\left(\beta_{2}-1\right) b \alpha_{1}^{\beta+3}}{6}+\frac{\gamma\left\{a_{1}+\alpha\left(a_{2}-a_{1}\right)\right\}\left(\beta_{2}-1\right) b \alpha_{1}^{\beta+3}}{6} \\
& +\frac{a_{3} \gamma b}{6}\left(1-\alpha_{1}^{\beta+3}\right)-\frac{\gamma b\left\{a_{3}-\alpha\left(a_{3}-a_{2}\right)\right\}\left(1-\alpha_{1}^{\beta+3}\right)}{6}, \\
& F_{2}=\frac{\left\{a_{3}-\alpha\left(a_{3}-a_{2}\right)\right\}}{b}\left(1-\alpha_{1}\right), \\
& G_{2}=\frac{a_{3} \gamma\left(\alpha_{1}^{\beta+1}-1\right)}{b(\beta+1)}+\frac{\left\{a_{3}-\alpha\left(a_{3}-a_{2}\right)\right\} \gamma}{b}\left(\alpha_{1}-1\right), \\
& H_{2}=\frac{\left\{a_{3}-\alpha\left(a_{3}-a_{2}\right)\right\}}{b^{2}}+\frac{\left(\beta_{2}-1\right)}{b^{2}}\left\{a_{1}+\alpha\left(a_{2}-a_{1}\right)\right\} \text {, } \\
& I_{2}=-\frac{\left\{a_{3}-\alpha\left(a_{3}-a_{2}\right)\right\}}{b^{2}} .
\end{aligned}
$$

\section{Acknowledgments}

The author is thankful to the honourable referee and the editor-in-chief for their constructive suggestions for preparing this final version of the paper. 


\section{References}

[1] W. A. Donaldson, "Inventory replenishment policy for a linear trend in demand: an analytical solution," Operational Research Quarterly, vol. 28, pp. 663-670, 1977.

[2] H. M. Wagner and T. M. Whitin, "Dynamic version of the economic lot size model," Management Science, vol. 5, pp. 89-96, 1958.

[3] E. A. Silver and H. C. Meal, "A simple modification of the EOQ for the case of a varying demand rate," Production Inventory Management, vol. 10, pp. 52-65, 1969.

[4] A. Mitra, J. F. Cox, and R. R. Jesse, "Note on determining order quantities with a linear trend in demand," Journal of the Operational Research Society, vol. 35, no. 2, pp. 141-144, 1984.

[5] M. Deb and K. Chaudhuri, "Note on the heuristic for replenishment of trended inventories considering shortages," Journal of the Operational Research Society, vol. 38, no. 5, pp. 459-463, 1987.

[6] U. Dave, "On a heuristic inventory replenishment rule for items with linearly increasing demand incorporating shortages," Journal of Operational Research Society, vol. 40, pp. 827-830, 1989.

[7] E. A. Silver, "Simple inventory replenishment decision rule for a linear trend in demand," Journal of the Operational Research Society, vol. 30, no. 1, pp. 71-75, 1979.

[8] U. Dave and L. K. Patel, “(T,Si) policy inventory model for deteriorating items with time proportional demand," Journal of the Operational Research Society, vol. 32, no. 2, pp. 137-142, 1981.

[9] R. S. Sachan, "On (T, Si) policy inventory model for deteriorating items with time proportional demand," Journal of the Operational Research Society, vol. 35, no. 11, pp. 1013-1019, 1984.

[10] H. Bahari-Kashani, "Replenishment schedule for deteriorating items with time proportional demand," Journal of operational Research Society, vol. 40, pp. 75-81, 1989.

[11] A. Goswami and K. Chaudhuri, "EOQ model for an inventory with a linear trend in demand and finite rate of replenishment considering shortages," International Journal of Systems Science, vol. 22, no. 1, pp. 181-187, 1991.

[12] M. Hariga, "EOQ model for deteriorating items with shortages and time-varying demand," Journal of the Operational Research Society, vol. 46, no. 3, pp. 398-404, 1995.

[13] L. A. Zadeh, "Fuzzy sets," Information and Computation, vol. 8, pp. 338-353, 1965.

[14] K. S. Park, "Fuzzy-set theoretic interpretation of economic order quantity," IEEE Transactions on Systems, Man and Cybernetics, vol. 17, no. 6, pp. 1082-1084, 1987.

[15] H. Ishii and T. Konno, "A stochastic inventory problem with fuzzy shortage cost," European Journal of Operational Research, vol. 106, no. 1, pp. 90-94, 1998.

[16] T. K. Roy and M. Maiti, "A fuzzy EOQ model with demand-dependent unit cost under limited storage capacity," European Journal of Operational Research, vol. 99, no. 2, pp. 425-432, 1997.

[17] S. C. Chang and J. S. Yao, "Economic reorder point for fuzzy backorder quantity," European Journal of Operational Research, vol. 109, no. 1, pp. 183-202, 1998.

[18] H. Katagiri and H. Ishii, "Some inventory problems with fuzzy shortage cost," Fuzzy Sets and Systems, vol. 111, no. 1, pp. 87-97, 2000.

[19] V. Krätschmer, "A unified approach to fuzzy random variables," Fuzzy Sets and Systems, vol. 123, no. 1, pp. 1-9, 2001.

[20] C. Kao and W.-K. Hsu, "A single-period inventory model with fuzzy demand," Computers $\mathcal{E}$ Mathematics with Applications, vol. 43, no. 6-7, pp. 841-848, 2002.

[21] H. A. Fergany and M. F. El-Wakeel, "Probabilistic single-item inventory problem with varying order cost under two linear constraints," Journal of the Egyptian Mathematical Society, vol. 12, no. 1, pp. 71-81, 2004.

[22] A. F. Hala and M. E. El-Saadani, "Constrained single period stochastic uniform inventory model with continuous distributions of demand and varying holding cost," Journal of Mathematics and Statistics, vol. 2, no. 1, pp. 334-338, 2006.

[23] L. Li, S. N. Kabadi, and K. P. K. Nair, "Fuzzy models for single-period inventory problem," Fuzzy Sets and Systems, vol. 132, no. 3, pp. 273-289, 2002.

[24] S. Banerjee and T. K. Roy, "Application of the intuitionistic fuzzy optimization in the constrained multi-objective stochastic inventory model," Journal of Technology, vol. 41, pp. 83-98, 2009.

[25] S. Banerjee and T. K. Roy, "Single and multi-objective stochastic inventory problems in fuzzy environment," Journal of Fuzzy Mathematics, vol. 16, no. 4, pp. 875-897, 2008.

[26] H. M. Lee and J. S. Yao, "Economic production quantity for fuzzy demand quantity and fuzzy production quantity," European Journal of Operational Research, vol. 109, no. 1, pp. 203-211, 1998.

[27] H. M. Lee and J. S. Yao, "Economic order quantity in fuzzy sense for inventory without backorder model," Fuzzy Sets and Systems, vol. 105, no. 1, pp. 13-31, 1999. 
[28] J. J. Buckley, Fuzzy Probabilities: New Approach and Application, Physica, Heidelberg, Germany, 2003.

[29] J. J. Buckley and E. Eslami, “Uncertain probabilities I: the continuous case," Soft Computing, vol. 7, pp. 500-505, 2003.

[30] J. J. Buckley and E. Eslami, “Uncertain probabilities II: the continuous case," Soft Computing, vol. 8, no. 3, pp. 193-199, 2004.

[31] J. J. Buckley and E. Eslami, "Uncertain probabilities III: the continuous case," Soft Computing, vol. 8, no. 3, pp. 200-206, 2004.

[32] C. M. Hwang and J. S. Yao, "Independent fuzzy random variables and their application," Fuzzy Sets and Systems, vol. 82, no. 3, pp. 335-350, 1996.

[33] A. Colubi, J. S. Domínguez-Menchero, M. López-Díaz, and D. A. Ralescu, “On the formalization of fuzzy random variables," Information Sciences, vol. 133, no. 1-2, pp. 3-6, 2001.

[34] M. K. Luhandjula, "Mathematical programming in the presence of fuzzy quantities and random variables," Journal of Fuzzy Mathematics, vol. 11, no. 1, pp. 27-39, 2003. 


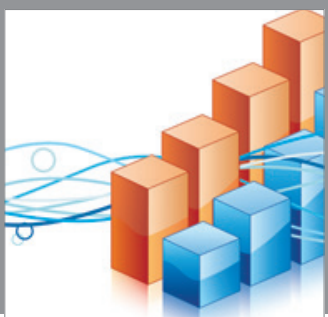

Advances in

Operations Research

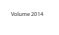

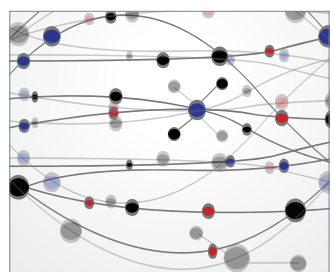

\section{The Scientific} World Journal
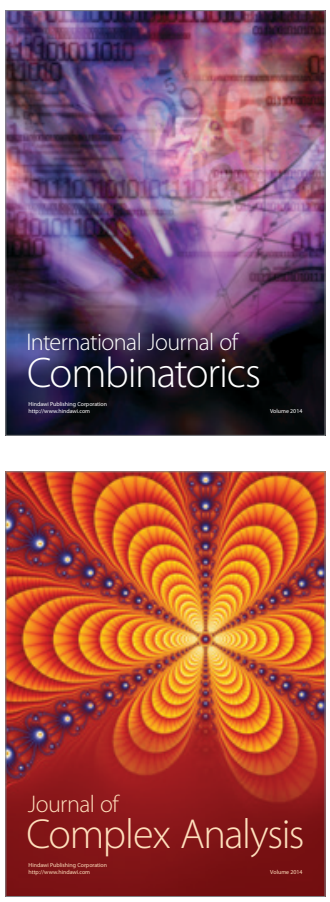

International Journal of

Mathematics and

Mathematical

Sciences
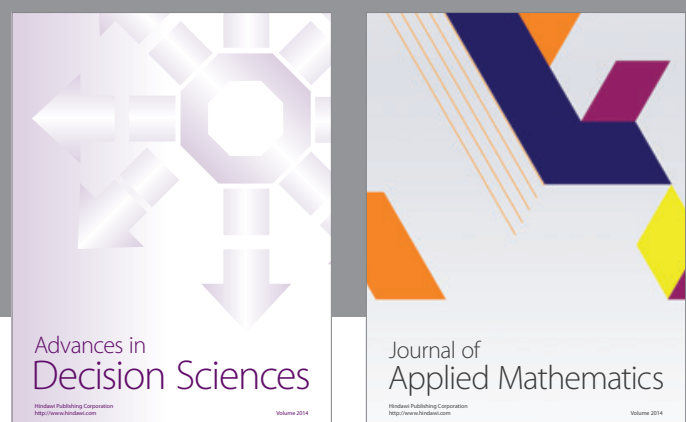

Journal of

Applied Mathematics
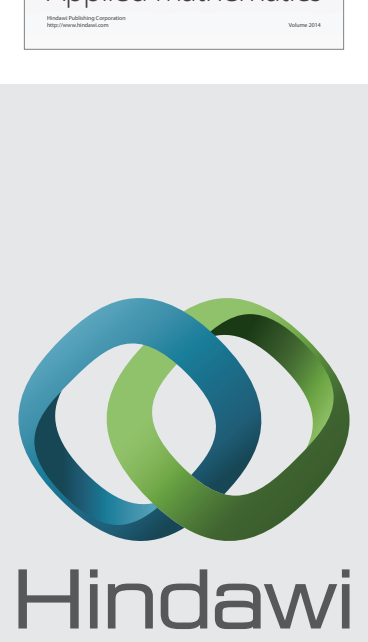

Submit your manuscripts at http://www.hindawi.com
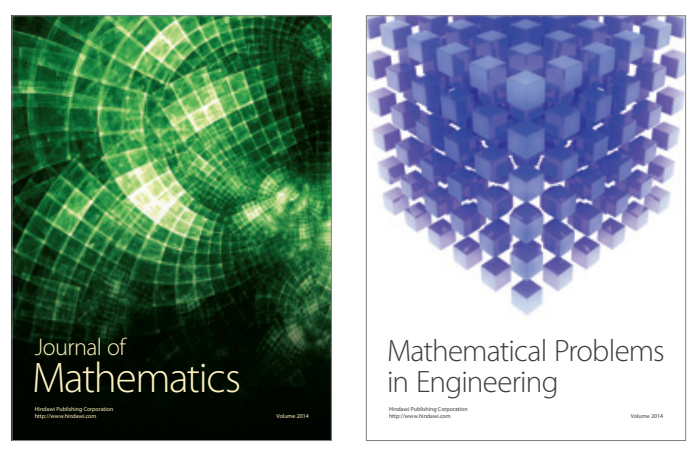

Mathematical Problems in Engineering
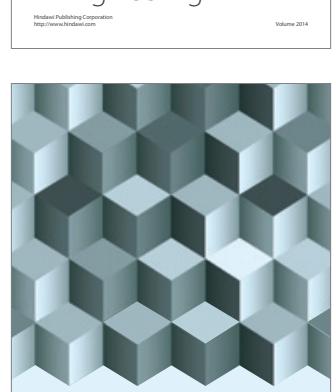

Journal of

Function Spaces
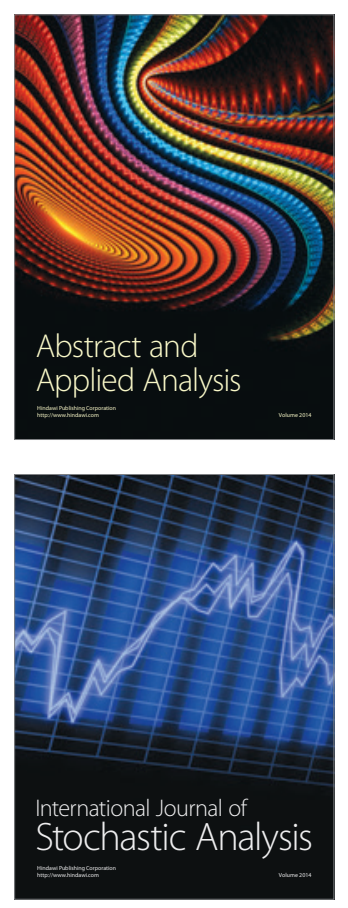

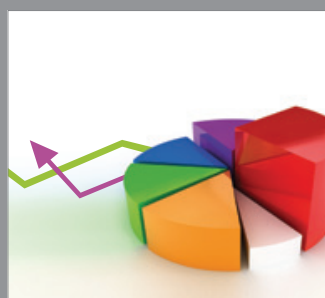

ournal of

Probability and Statistics

Promensencen
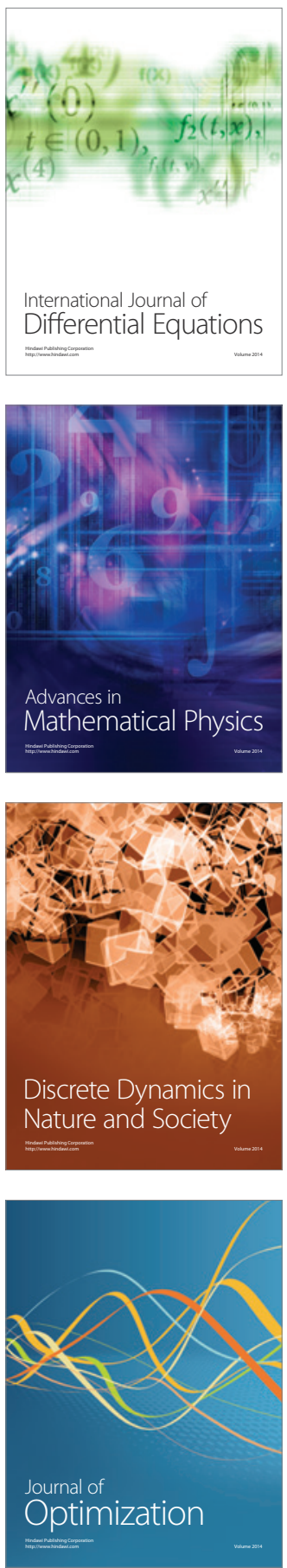